\title{
The World Health Organisation's Rationale for Physical Activity: a Philosophical Critique
}

\author{
Andrew Bloodworth, Mike McNamee
}

University of Wales Swansea, School of Health Science, Centre for Philosophy, Humanities and Law in Healthcare, University of Wales Swansea, UK

ABSTRACT

The World Health Organisation's rationale for physical activity draws heavily on scientific evidence regarding disease and obesity. Greater philosophical reflection on such concepts, along with a recognition that supposed scientific facts are rarely value-free, allow for a more positive and considered argument for physical activity and its benefits. Olympism, Olympic culture, sports education, pedagogy of sport

KEYWORDS well-being, health, disease, obesity

\section{Introduction}

The precise nature of the relationship between physical activity and health is disputable in a number of ways. One might question the kinds of physical activity under discussion, how long it should be undertaken for, or with what intensity. Equally one might challenge the conception of health adopted for the relationship to be tested or evaluated. Questions such as these speak to the rationale for physical activity within any kind of health-promoting arena. The leading international agency for health, the World Health Organisation (WHO), considers physical activity largely as a means for the 'prevention of chronic non-communicable diseases and Health Promotion' (Puska 2004: 17). Extensive research has been conducted in an attempt to establish a firm scientific basis for associations between physical activity and prevention of specific diseases (Vuori 2004). In this sense the rationale for physical activity presented by the WHO is in fairly negative terms. In contrast to the WHO rationale we shall argue that concepts such as disease and illness are only intelligible when considered in association with ideas of human needs, what we need to do, how we need to function in order to live a good life. They are not purely natural or physical concepts, but act powerfully to indicate how we want to live our lives and what we require to do this. This form of philosophical analysis may help us to reframe the benefits of physical activity in more positive terms.

Critical consideration of the concepts of 'disease' and 'illness' may encourage us to reflect upon the necessary requirements of good living, but we should not be seduced into thinking that an absence of disease or illness, or even being in good health is sufficient for us to equate that mode of existence with well-being or a good life. We develop a critique of the limitations of the WHO rationale based on the insight that its focus upon disease and illness, fails to recognise or appreciate the ways in which physical activity may impact upon our well-being. 
The two main aims of this critique should be made clear. The first is to illuminate the 'entanglement of fact and value' (Putnam 2002: 3) within concepts such as disease and illness, and more obviously obesity. This facilitates the exploration of the benefits of physical activity in terms of human need, a route to a more positive argument for the benefits of physical activity. The recognition that science is not value-free should not be seen as a destructive effort, eliminating any potential for objectivity, or reasonable argument when we consider the benefits of physical activity or whether we should exercise. The underlying epistemological perspective of this essay is that we can achieve some objectivity in our account of the value of physical activity and its relation to health, that values are not beyond the realm of rational argument and that concepts such as well-being are justifiable ground for rational deliberation. This perspective is seen more clearly within the second aim of the paper, to illustrate other potential ways in which physical activity may impact upon our well-being without reference to its capacity to prevent disease. Our argument stands in contrast to the fashionable and dominant idea that the domain of well-being is entirely subjective.

Prior to this main argument, however, we will begin with some important definitions and introductions to concepts that will be prominent throughout this paper.

\section{What counts as "physical activity" and "health"? The WHO position}

Physical Activity is interpreted very widely within the WHO's rationale for physical activity, when referring to the recommended amount of physical activity per day it is stated:

This level of activity can be reached through a broad range of appropriate and enjoyable physical activities and body movements in people's daily lives, such as walking to work, climbing stairs, gardening, dancing, as well as a variety of leisure and recreational sports.

$$
\text { (WHO 2003c: 3). }
$$

Defining physical activity in this broad manner makes good sense. Those who are less 'sporty' have an ample range of alternative activities with which to make up the recommended thirty minutes of moderate physical activity per day. There are however, difficulties with a definition of such breadth. A rationale (such as the WHO's) that makes extensive reference to the physical benefits of physical activity, that does not seek to differentiate between activities such as cleaning or walking to work and sport or exercise, may under-emphasise the benefits the latter activities offer, extending beyond the physical domain. Recreational sport or exercise may offer social opportunities, challenge, or enhance perceptions of control, benefits less evident in the more basic forms of physical activity, despite the similar energy expenditure.

The WHO defines health as "a state of complete physical, mental and social well-being and not simply the absence of disease or infirmity" (WHO 1948). It is somewhat paradoxical that this definition insists upon the positive aspects of health when the rationale for physical activity is in mainly negative terms. This extremely broad definition of health also suffers from the WHO's reluctance to properly conceptualise well-being. Well-being is referred to a number of times within the rationale for physical activity, but not defined, although there is often alignment with the psychological benefits of exercise such as improved mood.

\section{The Fact/Value Dichotomy}

While they are often held up as opposites, especially by positivistically inclined scientists (McNamee, 2005), distinctions made between judgements of fact and judgements of value need not 
necessarily be of great significance. Indeed they need not have "any implications at all as to whether value judgements can or cannot be true or false, justified or unjustified" (Putnam 2002: 61). It is the dichotomy between fact and value of which Putnam (2002) provides an extensive critique and, it will be claimed, is of evidence in the rationale for physical activity provided by the WHO.

The idea that "value judgements are subjective" is a piece of philosophy that has gradually come to be accepted by many people as if it were common sense. In the hands of sophisticated thinkers this idea can be and has been developed in different ways. The ones I shall be concerned with hold that "statements of fact" are capable of being "objectively true" and capable, as well, of being "objectively warranted," while value judgements, according to these thinkers, are incapable of object truth and objective warrant. Value judgements, according to the most extreme proponents of a sharp "fact/value" dichotomy, are completely outside the sphere of reason.

(Putnam 2002: 1).

This opening statement of Putnam's text illustrates the stance held by those in support of the dichotomy. In following Putnam's account of the history of the dichotomy it becomes apparent that the notion of fact (and its separation from the sphere of value) has been defended (in a somewhat dubious fashion) on scientific grounds. Science provides a value-free route to objectivity, entirely separate from the ethical domain. It will be the contention here that many of the 'facts' employed in science are entangled, to use the same specific terminology as Putnam, with values. Disease and illness, it is suggested, are concepts bound up with ideas about what we need in order to live a good life. This reflects Putnam's argument that "the picture of our language in which nothing can be both a fact and value-laden is wholly inadequate and that an enormous amount of our descriptive vocabulary is and has to be "entangled"” (Putnam 2002: 62).

Excluding reason over values seems at odds with our own thoughts and conversations. Griffin (1986; 1996) describes how an individual may criticise their own desires, asking, having observed someone else's genuine accomplishment, whether their own more happy-go-lucky existence is lacking something because of the absence of this value. This reflection over what is good for ourselves and others suggests that reasoning is not restricted to the scientific domain but that the way we think about our well-being reflects our search for some degree of objectivity in the ethical domain as well. We question whether something is of value to someone regardless as to their endorsement, indeed we question our own decisions and desires. The answers may not be easily accessible as Putnam (2002) is at pains to point out that the search for an absolute truth separate from any perspective is unachievable in science or anywhere else, but this need not, and it seems if we examine the ways in which we reason over value, does not stop us reflecting on ethical issues, including those concerning well-being. This is pretty good news for anyone hoping to build a coherent rationale in the area of physical activity since it will properly appeal to some concept of well-being and its constituents. If we were to accept the fact/value dichotomy this form of reflection would be blocked, providing a somewhat impoverished rationale, unable to lay claim to any form of objectivity regarding what is good for us apart from evidence provided by the sciences. 


\section{The World Health Organisation's Rationale for Physical Activity}

\section{DEATH, DISEASE AND PHYSICAL AILMENTS}

The most prominent argument for regular physical activity within the WHO rationale is that concerning the negative sense of health, i.e. the prevention of unwanted diseases and symptoms. The World Health Organisation have produced a summary of the benefits of physical activity (see both WHO 2003c and fact sheet Benefits of physical activity (WHO 2005a)), of which a large percentage of the bullet points concern this aspect. Those relevant to this section are shown below.

Figure 1. Benefits of physical activity (taken from WHO 2003c Health Development Through Physical Activity and Sport page 3).

- reduces the risk of dying prematurely

- reduces the risk of dying from heart disease or a stroke, which are responsible for one-third of all deaths

- reduces the risk of developing heart disease, colon cancer and type 2 diabetes

- helps to prevent/reduce hypertension, which affects one-fifth of the world's adult population

- helps to prevent/reduce osteoporosis, reducing the risk of hip fracture in women

- reduces the risk of developing lower back pain can help in the management of painful conditions, like back or knee pain

- helps build and maintain healthy bones, muscles, and joints and makes people with chronic disabling conditions improve their stamina

All but the last point concern the reduction or prevention of unwanted physical symptoms, disease or death. The apparent universality of these conditions appears to provide the rationale with a degree of universality itself. It is suggested that the drive to increase physical activity should apply as much to developing countries citing both growing inactivity (WHO 2002) in developing countries and that the majority of deaths from non-communicable diseases occur in developing countries (WHO 2002). If physical activity can play a part in the reduction of disease and premature death, this surely forms a particularly strong aspect of the rationale.

We must insist, however, that concepts such as 'disease' and 'illness' are not accepted as natural categories. Not only is this inaccurate (they are highly contested categories ${ }^{1}$ ), but it also prevents further analysis of these concepts. To say that physical activity prevents disease does not necessarily get to the bottom of what is valuable to us. Disease itself is not a value-free concept, and it may well be beneficial for us to debate the values that its conceptualisation throws up. Sedgwick (1982) provides a response to claims that disease and illness are natural facts:

What, they will protest, are there no diseases in nature? Are there not infectious and contagious bacilli? Are there not definite and objective lesions in the cellular structures of the human body? Are there not fractures of bones, the fatal ruptures of tissues, the malignant multiplications of tumorous growths? Are not these, surely, events of nature? Yet these, as natural events do not constitute illnesses, sicknesses, or diseases prior to the human social meanings we attach to them. The fracture of a septuagenarian's femur has, within the world of nature, no more significance than the snapping of an autumn leaf from its twig

(Sedgwick 1982: 30).

\footnotetext{
${ }^{1}$ See for example Richman (2004).
} 
If these natural events are insignificant in themselves, why do we attribute such significance to them? Sedgwick suggests that we "consider as 'illnesses' or 'diseases' those natural circumstances which precipitate death (or the failure to function according to certain values)" (Sedgwick 1982: 30-1). So we categorise something as a disease or illness, this line of argument suggests, if we associate it with death or the prevention of functions, central functions we could hypothesise bearing in mind the almost universality of certain disease classifications, and the importance that we attribute to them.

Let us sum up so far. The WHO has provided a rationale for physical activity mainly in terms of the prevention of disease. It refers to heart disease, hypertension, osteoporosis and back pain amongst other conditions. These conditions, it is argued, are not of natural significance, we have attributed meaning to such diseases and illnesses because of their impact upon certain central functions, functions we may presume to be central to our leading a good life or achieving well-being. The WHO rationale for physical activity does not explicitly recognise this entanglement, seeming to presume the apparent obvious nature of the 'badness' of the diseases and illnesses discussed. Some may concur with this, perhaps suggesting that these illnesses are so entangled, so intertwined with the negative social meaning to which we ascribe them that there is no room for argument. This seems a defensible retort. But, our reply goes, recognising this entanglement is the first step along the road to a positive argument for physical activity. It shows how physical activity impacts upon central human needs. There may be potential here for extension. We may be able, after extensive reflection, to suggest certain central human needs, and indeed recognise how physical activity may impact upon these. Illness and disease certainly reflect the most serious need frustration (von Wright 1996), but an argument for physical activity couched in the needs that it satisfies could investigate a greater range of its benefits, not just in preventing disease and illness but in positively effecting the satisfaction of needs above the illness threshold. This would expand upon the WHO's final statement in the bullet points above, referring to healthy bones and stamina. Perhaps we could understand some sort of mobility or locomotion as a central need or function (see Schramme 2002), and instead of seeing physical activity only as preventing diseases that frustrate this need, understand how physical activity can promote this function, improving our strength and flexibility, and therefore our ability to conduct daily tasks, playing with the children, gardening, having the necessary strength to get out of a chair with ease. This would surely be a worthwhile step, one that would take us from a rationale for how physical activity can stop us becoming something (ill or diseased), to how it can contribute to the foundations for our living any sort of good life.

This approach relates to conceptions of health such as Nordenfelt's who understands health in terms of the ability to achieve vital goals, those goals "necessary and together sufficient for minimal happiness" (1995: 90). Health is understood with reference to a concept of happiness, or ideas of valuable ends. This would not only enable us to understand disease and illness as a frustration of these ends, but to consider the value of sport and exercise in terms of vital goals, providing a potentially more convincing and positive rationale.

Where have we arrived at as a result of the foregoing discussion? A number of philosophers have incorporated health as a central aspect of welfare (Rescher 1972) but insisted that welfare is a fairly minimal concept, not reaching the breadth of well-being. Von Wright has stated that the 'privative notion of health' (von Wright 1996: 62) 'is conceptually allied to the needs and wants of beings and to the notions of the beneficial and the harmful' (von Wright 1996: 62), continuing on to associate pleasure with a more positive concept of health. With regard to our critique of the rationale for physical activity provided by the WHO, the perspectives of both von Wright and Rescher resonate to a degree. Considerations of disease and illness, and of the needs that such concepts imply will not necessarily exhaust our conception of the good life. There is a distinction to be made between those factors necessary for us to live a good life, or have a life with a decent level of well-being (for example 
the absence of disease or illness), and those factors or values that make up a good life. Griffin, for example, considers needs-based accounts of well-being to fail to get to the bottom of what is valuable to us, imposing an unjustifiable hierarchy between needs and desires (Griffin 1986). Amongst Griffin's suggested universal prudential values ("everything that makes a life good simply for the person living it' (Griffin 1996: 19) are accomplishment and enjoyment, neither of these it seems are likely to result from a philosophical examination of the concepts of disease and illness.

Our brief analysis of the concepts of disease and illness may indicate a more positive rationale for physical activity, how it may help to satisfy certain central needs. These needs, however, may not prove sufficient for a good life, or a life of well-being. Although the question of the adequacy of needs accounts as theories of well-being requires further debate, ${ }^{2}$ this need not be resolved here. The WHO have not proposed a theory of well-being, but indicated in some depth the potential of physical activity to prevent disease. The diseases incorporated within the rationale may well be entangled with certain needs, and basic functions, but these will not necessarily encompass all those functions and needs required for a good life, they may be incomplete as far as a needs-based account of well-being goes, apart from the discussion as to whether needs-based accounts are suitable theories at all.

Indeed, it seems likely that the WHO have omitted or ignored certain benefits of physical activity, or at least failed to give such benefits adequate emphasis. The rationale itself recognises some benefits of physical activity that extend beyond both the prevention of disease and illness and the psychological benefits that will be addressed later. These benefits are not included in the bullet points summarising the overall benefits, but mentioned in the discussions of the benefits of physical activity for particular populations. For "Children and Young People" (WHO 2003c: 4) it is recognised that 'Engagement in play and sports gives young people opportunities for natural self-expression, selfconfidence, relief of tension, achievement, social interaction and integration as well as for learning the spirit of solidarity and fair play' (WHO 2003c: 4).

There is no doubt that the rationale considers these benefits valuable. These benefits, however, are not included in the bulk of the rationale that appears to focus upon the physical aspects of health and obesity. As a result they are easy to miss or pass over, and one can easily envisage a policy maker persuaded by the rationale concentrating more upon the physical benefits, and prescriptions for 30 minutes exercise a day without concerning themselves too much with what else physical activity might promote. There is also the additional argument that physical activity that has at its core, social aspects or achievement, for example, is likely to prove more rewarding and thus encourage further participation.

Again, the WHO has missed an opportunity for extension of their rationale for physical activity. These benefits of physical activity, the social aspects, being able to express yourself, achievement (perhaps related to Griffin's idea of the sense of accomplishment), are given little emphasis within a rationale that focuses upon the prevention of disease. There is an argument here for a more comprehensive and integrated argument for how physical activity contributes to well-being. This would of course require a developed concept of well-being, a concept that extends beyond conclusions that well-being is entirely subjective, a result of desire satisfaction or pleasure, ${ }^{3}$ and concludes that certain values are necessary to our leading a good life. These values may include that which the WHO have suggested that sport (in the best instances) may offer, accomplishment, expression, social integration. This would of course require a sizable epistemological shift, renouncing the primacy of the scientific data, not only recognising that values are inherent in disease and illness

\footnotetext{
${ }^{2}$ See Griffin 1986, chapter III for an argument against the basic needs account as an adequate theory of wellbeing.

${ }^{3}$ See Griffin 1986 chapter I for a discussion of the inadequacy of the unrefined versions of these theories.
} 
for example, but also suggesting that there is scope for an objective conception of value, that science does not provide the only route toward objectivity. An objective conception of well-being would be enabled through such a conclusion and this does not seem so far fetched when we consider for example that we are often mistaken in the judgement of our own well-being, and we often question whether people are really aware of what is best for them. Understanding the values that inform such conclusions may help us to understand how physical activity may contribute to our lives in a significant way apart from its influence upon disease and illness.

A rationale for physical activity that places greater emphasis on those aspects of our wellbeing less related to the negative sense of health has further implications. Establishing that certain values such as accomplishment or enjoyment (Griffin 1986), or indeed those suggested by the WHO itself (self-expression, achievement, social interaction) are constitutive of our well-being provides a strong case for the forms of physical activity most likely to instantiate these values. This may entail an argument for more meaningful forms of physical activity, sporting activities, and active types of play offer more in the way of these values than opting to take the stairs rather than the escalator. The broad definition of physical activity employed by the WHO may have its advantages, as we have discussed, but greater reflection on what makes up our well-being would help us develop ideas about the structure physical activity should take to best enhance our well-being.

The first part of this section argued for the entanglement of concepts such as disease and illness (central to the WHO rationale for physical activity), with notions of basic human need and central human functions. The WHO concentrates on physical activity and prevention of disease, but if this necessarily entails considerations of human need, or central functions, there is room here for a more positive argument for physical activity, considering its benefits more directly in these terms. The analysis of disease and illness will not encompass the whole of well-being, sport and exercise in its more meaningful forms in particular, are of value to us in further ways. The WHO recognises these ways but is reluctant to incorporate these benefits in a more formal framework, primacy is given to parts of the rationale with a more scientific leaning. It may be, however, that certain values are integral, indeed constitutive of our well-being, conceiving of well-being in this fashion may help us to suggest ways in which sport, exercise and physical activities enhance our well-being beyond disease prevention.

\section{Physical activity and Obesity}

A further benefit of physical activity indicated by the WHO concerns obesity. We are told in the 'Health and Development Through Physical Activity and Sport' (WHO 2003c) document that "regular physical activity:

helps control weight and lower the risk of becoming obese" (WHO 2003c: 3).

With reference to women in particular we are told:

"Regular physical activity combined, with adequate diet has shown to be one of the most effective means of controlling mild to moderate obesity and maintaining an ideal body weight in women" (WHO 2005b).

The WHO rationale for physical activity appears to embrace the current view of obesity as an epidemic. Such talk surrounding obesity is not unusual, the popular press contains a great deal of reference to the topic and indeed to the urgency of the problem, which according to such sources, cannot be overstated. 
Evans (2003) has provided comprehensive criticism of this stance, pointing out that obesity is a constructed concept, not a physical one:

First, while 'fat' can be considered, at least in part, a physical/visceral condition, 'weight', 'overweight' and 'obesity' cannot. They are each a 'social arbitrary', measured constructions in the thinking of someone (e.g. researchers or the medical profession, or the insurance companies for whom indexes of the body mass index (BMI) type were originally designed).

\section{(Evans 2003: 88)}

Evans goes on to state that knowledge with regard to health consequences of obesity is uncertain, indeed the literature surrounding the whole area seems somewhat uncertain, for example the criteria for classification of obesity varies (Evans 2003). Evans does not seek to dismiss obesity as a health problem out of hand, but urges proper reflection on the uncertainties the data present; the WHO, states Evans, is one of the organisations that fail to recognise this uncertainty.

What is most concerning about this 'pushing' of the obesity line, despite the uncertainty regarding the evidence, are the consequences for those individuals which such campaigns are aimed at. We have noted the WHO's statement that exercise can help women achieve ideal body shape. The obesity research with all the doubt surrounding it cannot help us establish what this ideal body shape is and why exactly this mysterious ideal would be beneficial, and the health consequences of 'obese' or 'overweight' remain uncertain (Evans 2003).

As in the case of disease, the WHO has paid limited attention to the concept of obesity, accepting it and the evidence associated with it at face value. The implications of this inadequate attention to obesity as a concept, though, are very different from those concerning disease. In the previous section we cited that the rationale was incomplete without recognition of how physical activity might help us function and promote basic needs such as mobility. The rationale was limited by a failure to recognise the entanglement of such values with the concept of disease. The implications stemming from the WHO's emphasis on obesity are different. The focus on body shape is misplaced and potentially very damaging bearing in mind the complexity and uncertainty that surrounds the association between size and health (Evans 2003). Evans cites that his arguments resonate with the 'Health at Any Size (HAAS) movement', a very different approach to that advocated by the WHO here and its emphasis on weight reduction.

The scientific data on the topic of obesity, or at least the conclusions that have been drawn from it, have been subject to extensive criticism. Recognition that obesity, despite its apparent scientific appearance, is certainly not value-free, would perhaps initiate a more critical look at the evidence and more considered policies resulting from this examination. Again, we should state that to recognise the infusion of a concept with values and to question scientific objectivity, need not lead to the abandonment of objectivity or reasoning in the area altogether. Putnam (2003) indicates that the search for absolute (i.e. non-perspectival or non-theory laden) knowledge is flawed altogether, but provides ample evidence for how fact and value are entangled in the ethical domain, including extensive reference to Sen's capabilities approach to the quality of life (see Putnam 2002). There seems to be no easy way of justifying how certain ethical viewpoints and indeed theories of well-being or quality of life offer something more than just someone's view point, but this need not mean we give up on the idea of objectivity altogether:

But recognizing that our judgements claim objective validity and recognizing that they are shaped by a particular culture and by a particular problematic situation are not incompatible. And this is true of scientific questions as well as ethical ones. The solution is neither to give up on the very possibility of rational discussion nor to seek an Archimedian point, an "absolute 
conception" outside of all contexts and problematic solutions, but - as Dewey taught his whole life long - to investigate and discuss and try things out cooperatively, democratically, and above all fallibilistically.

(Putnam 2002: 45)

With reference to obesity and the rationale for exercise, to recognise the concept as shaped by culture need not render it incapable of delivering any form of objectivity. But there seems to have been little of the investigation and discussion that Putnam speaks of in the WHO's use of the concept. Increased discussion, such as that utilised by those such as Evans who attack the current use of the concept, may help develop our ideas on the issue and indeed move us closer to an objective idea of what obesity is and how it impacts upon our lives. This critical thinking would no doubt employ the concepts of 'need' and 'well-being' referred to in the last section, providing an interesting way of testing where obesity may genuinely have negative impact on the central functions of our lives, and where its impact upon these areas may be questioned, for example where the emphasis on ideal body shape has more to do with certain approaches to beauty than with maintaining central human functions and basic human needs.

\section{Physical activity and psychological well-being}

Physical activity, states the WHO, promotes "psychological well-being, reduces stress, anxiety and depression" (WHO 2003c: 3). This draws upon the work of exercise psychologists such as Biddle and colleagues (2000). Such work is certainly valuable: increases in mood and self-esteem as well as decreases in negative symptoms such as anxiety and depression are not only important consequences, but also valued motivators for continued participation.

In emphasising the psychological aspects of well-being, we can again trace an implicit acceptance of the fact/value dichotomy, this time not concerned with the assertion of scientific objectivity, but with the subjectivity of value, and indeed of well-being altogether. An emphasis on subjective aspects such as stress and anxiety is in line with the predominant approach to well-being in the psychological literature, ${ }^{4}$ subjective well-being, said to comprise life satisfaction and the balance of positive-negative affect (Diener and Lucas 1999). In focusing upon psychological aspects of wellbeing the WHO can be said to be maintaining a pretence of neutrality emphasising the supposed noncontroversial concepts of anxiety and stress, but making little further addition to our understanding of what well-being is.

Further evidence for this reluctance on the part of the WHO to reason or debate over the constituents of well-being has already been given. The rationale for physical activity attributes a primacy to the scientific data, and whilst it does refer to a number of other benefits of physical activity, self-expression, achievement, the social aspects, and the psychological benefits, these benefits are left somewhat free-floating. An extension of the rationale would be to suggest how these aspects are constitutive of our well-being, the epistemological shift discussed earlier, allowing for an objective conception of value, ensuring that these benefits of physical activity are on a level footing with the physical benefits stemming from the scientific aspect of the rationale.

The WHO though appears to maintain this image of a neutral stance regarding the constituents

\footnotetext{
${ }^{4}$ Although we should note both the work Ryff and Singer along with Self Determination theorists as departing from the subjective well-being. (see Ryff and Singer 1998 on positive health, and for 'A Review of Research on Hedonic and Eudaimonic Well-Being' see Ryan and Deci (2001), prominent self-determination theorists, providing ample indication of how well-being is conceptualised from a self determination theory perspective.
} 
of well-being, concentrating on the psychological states that may accompany any number of values. Putnam (2002) has illustrated how attempts to employ a neutral concept of utility within welfare economics were doomed, and it seems that psychological concepts of well-being are also fatally flawed.

First there is a privileging of certain psychological states, pleasure for instance, or the absence of states such as anxiety, stress, or depression. These values need not exhaust our conception of wellbeing, indeed an over-emphasis of these factors would lead to a somewhat impoverished conception. Sporting participation in particular may include moments of anxiety or stress, and many physical activities are not pleasurable in the conventional sense, but our continued participation indicates that we think they enhance our well-being. Conversely activities that are pleasurable do not always enhance our well-being. Loumidis and Wells (2001) have examined the use of exercise in avoidance or emotion-focused coping strategies.

In that way although individuals might 'feel better' emotionally following the implementation of an emotion-focused or avoidance strategy, in the long term the core and fundamental aetiological factors of emotional distress are not dealt with. In such a case they might continue holding negative beliefs such as 'I am worthless' or 'If I do not exercise I will become sexually unattractive' and continue to be vulnerable for future distress.

(Loumidis and Wells 2001: 422).

Here exercise appears despite its initial positive impact to be masking deeper problems, and its contribution to well-being could be questioned as participation seems only to be reinforcing negative self-perceptions.

Subjective conceptions of well-being, emphasising reduced anxiety, stress, and depression (WHO 2003c) and enhanced mood are deeply flawed. What we find pleasurable might not always be good for us, we can be mistaken with regard to our own well-being. If we are to provide an adequate description of how, or indeed in what instances exercise does enhance our well-being, and therefore a fully considered rationale for physical activity, we must account for such mistakes and this may require reflection upon certain values that may be constitutive of our well-being, desires for which we could describe as informed ${ }^{5}$. This would require a rejection of the fact/value dichotomy, in particular a rejection of the subjectivity of value, considering certain values to be make up our well-being (perhaps even universal values such as those suggested by Griffin 1986), and championing those forms of physical activity that instantiate such values. ${ }^{6}$

\section{Conclusion}

An implicit adherence to the fact/value dichotomy, and an uncritical acceptance of scientific data, has limited the attempts of the WHO to provide an adequate rationale for the value of physical activity. Positivistic attempts by exercise and sports scientists, moreover, offer little assistance. A failure to recognise how a concept such as disease is entangled with ideas of human need and function, has resulted in the failure to recognise how physical activity's potential to reduce disease also implies a more positive conclusion regarding how physical activity may satisfy basic human needs and promote central functions. The failure to critically evaluate the literature surrounding obesity has led to

\footnotetext{
${ }^{5}$ The process of recognising the fallibility of our actual desires, moving to correct these desires, and finding that this requires a more objective conception of prudential value is exactly that undertaken by Griffin 1986.

${ }^{6}$ For a fuller critique of exercise and sports psychologists utilisation of subjective models of well being see Bloodworth and McNamee (2007).
} 
an unfounded and potentially damaging emphasis on the potential of physical activity to adjust body size and shape. Finally, the emphasis on the psychological aspects of our well-being appears to stem from an underpinning assumption regarding the subjectivity of value ${ }^{7}$, but an acceptance of these foundations leads to some counterintuitive conclusions regarding exercise and its contribution to wellbeing. Exercise may indeed increase well-being, but the psychological aspects do not exhaust this concept, indeed reflection on other values that are constitutive of our well-being may help us understand when pleasure and the satisfaction of our actual desires may not provide the best indicator of well-being, and conversely of when they may be suitably informed.

\section{REFERENCES}

Biddle, S., Fox K., \& Boutcher S.H. (Eds.) (2000). Physical Activity and Psychological Well-Being. London: Routledge.

Bloodworth, A. and McNamee, M. J. (2007). Conceptions of well-being in the psychology and exercise psychology literature: A philosophical critique. Health Care Analysis, 15, (2):107-21.

Diener, E. \& Lucas R.E. (1999). Personality and Subjective Well-Being. In D. Kahneman, E. Diener, N. Schwarz (Eds.). Well-Being: The Foundations of Hedonic Psychology (pp. 213-229). New York: Russell Sage Foundation.

Evans, J. (2003). Physical education and health: a polemic or 'let them eat cake! European Physical Education Review, 9(1): 87-101.

Griffin, J. (1996). Value Judgement Improving Our Ethical Beliefs. Oxford: Clarendon Press.

Griffin, J. (1986). Well-Being Its Meaning Measurement and Moral Importance. Oxford: Clarendon Press.

Loumidis, K \& Wells, A (2001). Exercising for the Wrong Reasons: Relationships Among Eating Disorder Beliefs, Dysfunctional Exercise Beliefs and Coping. Clinical Psychology and Psychotherapy, 8: 416-23.

Nordenfelt, L. (1995). On the nature of health: an action theoretic approach. Dordrecht: Kluwer.

Puska, P. (2004). 'Foreward' in (Eds.) P. Oja \& J. Borms, Perspectives: Health Enhancing Physical Activity (pp. 17-19). Oxford: Meyer \& Meyer Sport (UK) Ltd.

Putnam, H. (2002). The Collapse of the Fact/Value Dichotomy and Other Essay. Cambridge, Massachusetts: Harvard University Press.

Rescher, N. (1972). Welfare The Social Issues in Philosophical Perspective. Pittsburgh: University of Pittsburgh Press.

Richman, K. (2004). Ethics and the metaphysics of medicine. Boston: MIT Press.

Ryan, R.M. \& Deci, E.L. (2001). On Happiness and Human Potentials: A Review of Research on Hedonic and Eudaimonic Well-Being. Annual Review of Psychology, 52: 141-66.

Schramme, T. (2002). 'Is it bad to have a disease?' In A. Gimmler, C. Lenk, G. Aumüller (Eds.). Health and Quality of Life (pp. 61-7). Hamburg, London: Lit Verlag Münster.

Sedgwick, P. (1982). Psycho Politics. London: Pluto Press Limited.

von Wright, G.H. (1996). The Varieties of Goodness. Bristol: Thoemmes Press.

Vuori, I. (2004). Inactivity as a Disease Risk and Health Benefits of increased Physical Activity. In P. Oja, J. Borms, (Eds.) Health Enhancing Physical Activity (pp 29-95). Oxford: Meyer \& Meyer Sport (UK) Ltd.

World Heath Organisation (1948). Preamble to the Constitution of the World Health Organization as adopted by the International Health Conference, New York, 19-22 June, 1946; signed on 22 July 1946 by the representatives

\footnotetext{
${ }^{7}$ This passage echoes arguments in Griffin (1996) chapter II, a critique of the taste model as an adequate explanation of prudential value, with reference to how we can be mistaken in our judgements of well-being. Griffin's own approach to prudential value seeks to dispense with another related dichotomy, that made between desire and understanding.
} 
of 61 States (Official Records of the World Health Organization, no. 2, p. 100) and entered into force on 7 April 1948.

World Health Organisation (2002). Myths about physical activity. Online. Available HTTP: http://www.who.int/docstore/world-health-day/2002/fact_sheets7.en.shtml (accessed 15 January 2006).

World Health Organisation (2003c). Health and Development Through Physical Activity and Sport, Online. Available HTTP: <http://whqlibdoc.who.int/hq/2003/WHO_NMH_NPH_PAH_03.2.pdf (accessed online 15 January 2006).

World Health Organisation (2005a). Benefits of Physical Activity. Online. Available HTTP: $<$ http://www.who.int/moveforhealth/advocacy/information_sheets/benefits/en/index.html (accessed online 15 January 2006).

World Health Organisation (2005b). Women and Physical Activity, Online. Available HTTP: $<\mathrm{http} / / / \mathrm{www}$. who.int/moveforhealth/advocacy/information_sheets/woman/en/index.html (accessed online 15 January 2006). 